\title{
Economic effects of Transmission Expansions: The case of the Regulated Contract Market in Chile
}

\author{
Javier Bustos-Salvagno \& Fernando Fuentes H.
}

\begin{abstract}
One of the main issues in deciding an expansion in power transmission capacity is the effect on generators' competition. This paper aims to perform an econometric estimation of the main determinants of long-term regulated contract prices from 2006 to 2014 and use the results to estimate the effect on market power reduction of an interconnection of the two main power systems in Chile.
\end{abstract}

Keywords - Power Transmission, Market Power, Contracts, Prices, Auctions, Chile.

\section{INTRODUCCION}

$\mathrm{L}$ as metodologías para analizar la conveniencia de una sexpansión en el sistema de transmisión de un país han cambiado al compás de la evolución de la regulación económica del sector eléctrico. Bajo el sistema de regulación de un monopolio, ya sea estatal o privado, vigente durante la mayor parte del siglo XX, el monopolista integrado verticalmente tenía la obligación de abastecer la demanda presente y futura. Durante la aplicación de este marco regulatorio, la expansión en transmisión se evaluaba simplemente a partir del análisis de costos de operación e inversión de las diferentes alternativas de expansión.

Con el cambio de paradigma regulatorio en el sistema eléctrico, el que incluyó la desintegración vertical entre los segmentos de generación, transmisión y distribución, se dio forma a un mercado competitivo en generación, mientras que la transmisión ha continuado mayoritariamente regulada como un monopolio natural. Esta liberalización ha implicado la presencia de una relación directa entre las características y evolución del sistema de transmisión y la dimensión competitiva del mercado de generación.

El objetivo de una expansión en transmisión es, ante todo, asegurar que existirá suficiente capacidad en las líneas para abastecer la demanda a partir de la generación que se desarrolle, de manera confiable y al menor costo posible. El criterio de decisión para dicha expansión, tomando en consideración que existen participantes del mercado con objetivos diversos y muchas veces opuestos, es un aspecto crítico en el desarrollo eficiente de los mercados eléctricos modernos. Como menciona $\mathrm{Wu}$ et al [1], el utilizar como criterio de decisión simplemente la opción de mínimo costo de operación e inversión, como se hacía en el marco del paradigma del mercado eléctrico integrado verticalmente, no sigue siendo viable.

J. Bustos-Salvagno, Profesor Adjunto, Universidad Alberto Hurtado, rjb92@georgetown.edu

F. Fuentes, Profesor Asistente, Universidad Alberto Hurtado, ffuentes@uahurtado.cl
Como indica de Nooij [2], lo antes indicado también es válido para el caso de las interconexiones entre sistemas, las cuales inicialmente se realizaron por motivos de seguridad de suministro, y también para permitir el comercio entre sistemas. Posteriormente, cuando los mercados eléctricos comenzaron a liberalizarse, la dimensión competitiva empezó a tener más relevancia, llegando a la situación actual en que incrementos relevantes en transmisión e interconexiones se fundamentan principalmente a partir de objetivos asociados al aumento en la competencia entre generadores (Borenstein et al. [3]). Esto se ve avalado por el hecho que si se produjera congestión en las líneas, los generadores localizados en determinadas áreas podrían ejercer poder de mercado local (Joskow y Tirole [4]). En el caso de sistemas con regulación de precios en el mercado spot, con un despacho centralizado a costo marginal declarado como en Chile, Arellano y Serra [5] encuentran que una expansión en transmisión también incrementa la eficiencia en términos de bienestar social, debido a un aumento en la inversión en generación de base, cuando ha existido una restricción de transmisión activa.

Cabe destacar que la discusión no se ha reservado al ámbito académico solamente. Los organismos regulatorios en países desarrollados comenzaron hace algunos años a incorporar metodologías de evaluación y planificación en transmisión que reflejan lo encontrado en la academia (European Commission [6]). En EEUU, el liderazgo en el tema lo ha presentado el operador independiente del sistema de California (CAISO). Desde el año 2004 existe una metodología comprensiva de evaluación, denominada "Transmission Economic Assessment Methodology" (TEAM), para la realización de un análisis costo-beneficio de una expansión en transmisión ${ }^{1}$. Su principal contribución ha sido el desarrollo de una metodología de identificación de beneficios, incorporando los precios de mercado de la electricidad en el análisis, más allá de la simple evaluación de costos de operación e inversión en distintos escenarios de transmisión.

En el caso de Chile, ha predominado el enfoque de costos, sin considerar el efecto en precios. Ello también ocurre en Sauma et al [10], que evalúa los aspectos privados y sociales de la integración de los sistemas eléctricos en la comunidad andina (Colombia, Ecuador, Perú, Bolivia y Chile), cuantificando como beneficios económicos por el lado de la oferta solamente aquellos medidos por la reducción del margen operacional (diferencia entre costo marginal del sistema y costo de operación), y por el lado de la demanda, los asociados a la reducción en el precio spot o costo marginal, el ahorro de costos del sistema e el impacto ambiental medido por disminución de emisiones contaminantes. Algo similar realiza Ulloa [11] al analizar la interconexión entre los

\footnotetext{
${ }^{1}$ CAISO [7], Wolak [8], Wolak [9].
} 
principales sistemas eléctricos chilenos (SIC y SING) y su impacto en emisiones de carbono. Sólo un estudio llevado a cabo por la Comisión Nacional de Energía (CNE [12]), sobre la evaluación de impactos económicos y sociales de la interconexión SIC y SING, consideró además de los efectos directos de la interconexión, los impactos sobre el nivel de competencia y riesgo en el mercado de generación. En dicho estudio se encontró evidencia de importantes efectos en bienestar social producto de la reducción en el poder de mercado en el segmento de generación.

Tanto en la literatura de mercados spot desregulados (i.e. California) como de despacho centralizado (con la excepción del estudio CNE antes citado), no se ha incluido una dimensión relevante del impacto de la competencia sobre el precio de contratos de suministro de largo plazo, o contratos forward ${ }^{2}$. En general esto no ha ocurrido debido a la ausencia de información pública sobre contratos de largo plazo. En este contexto, el objetivo del presente trabajo es plantear una metodología de estimación del efecto de una expansión relevante en transmisión (por la vía de una interconexión entre sistemas) sobre el precio promedio de contratos, y aplicarla a datos disponibles del mercado de contratos regulados en Chile. Esto es un aporte la literatura de decisiones de expansión en transmisión, así como en competencia de mercados eléctricos ${ }^{3}$.

El trabajo se estructura de la siguiente forma. En la sección II se describe brevemente el funcionamiento del mercado eléctrico chileno. En la sección III se desarrolla la metodología aplicada en el trabajo y se entregan antecedentes sobre los datos utilizados. En la sección IV y V se presentan los resultados econométricos y las proyecciones bajo escenarios con y sin interconexión. En la sección VI se presentan las conclusiones.

\section{MERCADO ELÉCTRICO CHILENO}

El marco regulatorio vigente en Chile quedó definido por la ley promulgada en el año 1982, la cual estableció la liberalización del mercado eléctrico, dando base a la existencia de un mercado spot y un mercado de contratos en el sector. En lo que se refiere al suministro de largo plazo, un generador tiene la opción de firmar contratos de suministro con distribuidoras eléctricas, para clientes regulados, o con grandes consumidores, denominados clientes libres de regulación ${ }^{4}$.

El mercado de contratos regulados, foco de la presente investigación, constituye cerca del $50 \%$ del suministro contratado de largo plazo en Chile (Bustos-Salvagno [14]). Cabe destacar que el mercado eléctrico chileno como conjunto está compuesto por cuatro sistemas que suministran energía eléctrica en el territorio continental. Los dos más importantes son el Sistema Interconectado Central (SIC) y el

\footnotetext{
${ }^{2}$ Los trabajos mencionados anteriormente usaban datos de precios de ventas de energía en bolsas del mercado spot.

${ }^{3}$ En Bustos-Salvagno y Fuentes [13] se realiza un estudio del mismo efecto sobre los precios contratos libres, los cuales son el resultado de acuerdos bilaterales y no de licitaciones, como en el presente documento.

${ }^{4}$ La legislación separó los clientes libres de los regulados de acuerdo a su potencia conectada, correspondiendo a aquellos con una potencia conectada inferior o igual a $2.000 \mathrm{~kW}$, o entre 500 y $2.000 \mathrm{~kW}$ que hayan optado por ser clientes regulados.
}

Sistema Interconectado del Norte Grande (SING). Mientras el SIC es un sistema hidrotérmico, el SING es un sistema mayoritariamente térmico.

Hasta el año 2004, Chile importaba todo su consumo de gas natural desde Argentina, país que decidió reducir sus exportaciones de dicho combustible progresivamente hasta interrumpirlas completamente. La crisis del Gas Argentino cambió sustancialmente el mercado de contratos eléctricos en Chile. Consistente con ello y como resultado de la incertidumbre existente respecto a la disponibilidad de gas natural, el Gobierno de Chile decide introducir un cambio regulatorio importante, conocido comúnmente como Ley Corta II. El objetivo fundamental de dicho cambio legal era incentivar la inversión en centrales que reemplazaran la capacidad de generación a gas natural existente. En este contexto, nadie estaba dispuesto a invertir en centrales a gas de ciclo combinado (por la ausencia del insumo básico), pero el mismo tiempo los generadores corrían riesgo al instalar centrales a carbón, y que las mismas no terminasen siendo rentables (en caso que Argentina retomara los envíos de gas).

La Ley Corta II introdujo un sistema de licitaciones abiertas y no discriminatorias por el suministro eléctrico para clientes regulados, a cargo de las empresas de distribución eléctrica. De esta manera se sustituyó el sistema tarifario vigente en base a precios de contratos regulados (fijados periódicamente por la autoridad), por un sistema de precios de subasta ${ }^{5}$. Asimismo, se estableció que cada distribuidora debía llamar a licitación con al menos 3 años de anticipación al inicio de suministro, diseñando los bloques de energía a licitar. Por su parte, cada generador podría ofrecer energía por todo un bloque o una cantidad limitada de sub-bloques. La adjudicación se realizaría a mínimo precio, con la presencia de un precio techo. Cabe relevar que bajo este nuevo esquema, el precio ofrecido y adjudicado se mantiene constante durante la duración del contrato, pero es indexado de acuerdo a ponderadores escogidos por el propio generador, usando índices de precios predefinidos por la autoridad regulatoria (CNE).

Las licitaciones, entonces, cambiaron la forma en que se determinan los precios de contratos regulados. Hasta el momento del cambio legal, los precios regulados o precios de nudo sólo consideraban costos esperados en el mercado spot de energía. En una licitación, en cambio, el precio ofertado incluye no sólo los costos esperados, sino también un margen de comercialización variable. Este margen está determinado por factores idiosincráticos al contrato (por ejemplo, duración, tamaño, sistema eléctrico, etc.) y factores propios del mercado (competencia y riesgo). Así, es esperable que los precios de contratos licitados sean mayores a los precios de nudo tradicionales. Esto no es necesariamente un problema, siempre y cuando el mercado sea competitivo y los riesgos de contratación tiendan a reducirse en el tiempo. A continuación se presenta una metodología econométrica que da cuenta de la formación de precios en estas licitaciones, la cual será empleada para efectos de medir el efecto de una interconexión entre los dos mayores sistemas del mercado eléctrico chileno.

\footnotetext{
${ }^{5}$ Dado que el precio regulado no estaba dando cuenta de los nuevos riesgos de contratación que implicaba la crisis del gas, era esperable que en las licitaciones se produjera una transferencia, a lo menos parcial, del riesgo de contratación a los clientes regulados.
} 


\section{METODOLOGIA}

El objetivo es estimar el impacto en el mercado de contratos regulados (sujetos a licitación) que traería una interconexión entre los sistemas SIC y SING. Para ello, metodológicamente se procederá de la siguiente manera. Primero se describe un modelo econométrico que permite encontrar los determinantes históricos de precios para contratos regulados. Segundo, a partir de los coeficientes estimados, se procederá a realizar proyecciones del efecto sobre los precios esperados de contratos regulados, por reducción del poder de mercado y reducción en el riesgo producto de una interconexión entre los sistemas SING y SIC.

Al existir licitaciones por suministro regulado que brindan información pública sobre todas las ofertas realizadas por un contrato, es apropiado utilizar un modelo que dé cuenta de ello. Fabra, Montero y Reguant [15] así como Varas y Rudnick [16] utilizan información de contratos efectivamente adjudicados, ya que realizan una estimación de determinantes de precios tanto libres como regulados. Aquí, al igual que en Bustos-Salvagno [14], se concentran los esfuerzos en el mercado regulado, por lo cual si se utiliza toda la información disponible de las ofertas realizadas en licitaciones para clientes regulados, contexto en el que es necesario usar metodologías aplicadas en la literatura de subastas.

El objetivo es determinar los parámetros estructurales del mercado de contratos para clientes bajo regulación de precios, utilizando regresiones econométricas con datos históricos de las licitaciones llevadas a cabo entre los años 2006 y 2014. Es interesante indicar que el uso de información histórica del mercado eléctrico para proyectar escenarios de precios ante cambios en la infraestructura de transmisión se basa en el espíritu de la metodología TEAM (Awad et al [17]).

La siguiente especificación econométrica está diseñada para estimar el margen sobre precios competitivos (Índice de Lerner), utilizando información de las ofertas llevadas a cabo.

$$
\begin{aligned}
\frac{P_{i t k}-C_{t k}}{P_{i t k}}= & \beta_{0}+\beta_{1} R S I_{i t k}+\beta_{2} D_{i}+\beta_{3} T_{i}+\beta_{4} A \tilde{n} o_{t}+ \\
& \beta_{5} \text { Sistema }_{k}+\beta_{6} V C m g_{t k}+\epsilon_{i t k}
\end{aligned}
$$

Donde:

$P_{i t k}=$ Precio de oferta del generador por el contrato o bloque $\mathrm{i}$, en el momento t y sistema $\mathrm{k}$.

$C_{t k}=$ Costo competitivo de proveer suministro en el sistema $\mathrm{k}$, al momento $\mathrm{t}$, que representa una estimación del precio de largo plazo, en función de las expectativas que se tengan al momento de la oferta respecto al costo de los insumos (combustibles) y la tecnología que con mayor probabilidad "marcará" el precio del mercado eléctrico $^{6}$. Desde el punto de vista del comportamiento, se esperaría que mientras mayor sea

\footnotetext{
${ }^{6}$ En este caso es posible utilizar el costo de desarrollo esperado o, alternativamente, el costo marginal esperado de sistema como variable. La elección finalmente hecha deberá considerar cuál de estos valores corresponde a una mejor predicción de la tendencia de precios en el futuro.
}

el costo de suministro de largo plazo, mayores serán los precios.

$D_{i}=$ Duración de Cada Contrato o bloque i. Desde el punto de vista del comportamiento, se esperaría que mientras mayor sea la duración del contrato, más atractivo sea dicho contrato para un generador competitivo y menores serán los precios ofrecidos. De hecho, un contrato más largo puede implicar un menor riesgo para el inversionista, por lo cual, se debiera cumplir que $\beta_{2}<0$.

$T_{i}=$ Tamaño del contrato i. Desde el punto de vista del comportamiento, se esperaría que mientras mayor sea el tamaño del contrato, menores sean los precios, en la medida que en dicha condición le permite un mejor poder de negociación frente al generador respectivo, por lo cual se debiera cumplir $\beta_{3}<0$.

$A \tilde{n} o_{t}=$ Variable Dummy $^{7}$ por cada año, la cual recoge posible cambios estructurales del mercado a través del tiempo, que pueden reflejar una tendencia no explicada por las otras variables incluidas.

Sistema $_{k}=$ Variable Dummy que identifica el sistema k, la cual refleja la posible diversidad en la determinación de los precios de los contratos en cada sistema eléctrico.

$V C M g_{t k}=$ Volatilidad del Costo Marginal del Sistema $\mathrm{k}$ en el momento t, medida como la desviación estándar. Representa el riesgo del generador, en tanto proveedor de suministro, al momento de contratar. Desde el punto de vista del comportamiento, se esperaría que mientras más grande sea esta varianza (mayor el riesgo), mayores serán los precios, por lo cual se debiera cumplir que $\beta_{6}>0$.

$R S I_{i t k}=$ Residual Supply Index o Índice de Oferta Residual del contrato o bloque i en el momento t y sistema $\mathrm{k}$, medido como:

$$
\begin{aligned}
R S I= & \frac{O T x B l o q-O M x B l o q}{T B l o q}, \text { donde: } \\
& \text { OTxBloq }=\text { Oferta total del bloque. } \\
& \text { OMxBloq }=\text { Oferta mayor del bloque } \\
& \text { TBloq }=\text { Tamaño total del bloque licitado }
\end{aligned}
$$

El RSI indica el poder de mercado en la licitación. Una disminución en el RSI indica que la oferta de mayor tamaño representa una porción más significativa de la oferta total. En

\footnotetext{
${ }^{7}$ Variable que asume los valores cero o uno, con el objetivo de identificar cambios en la relación que se está midiendo econométricamente.

${ }^{8}$ Nótese que la variable TBloq no necesariamente coincide con la variable OTxBloq, ya que la primera representa el tamaño del bloque y la segunda la suma total de las ofertas recibidas en el proceso de licitación.
} 
términos de los signos esperados se considera razonable que mientras mayor sea RSI menor sea el poder de mercado, dado que la oferta de mayor tamaño representa una porción menor de la oferta total. En tal caso, el margen de ganancia extranormal sería menor (es decir, $\frac{P-C}{P}$ ), con lo cual $\beta_{1}<0$.

Para llevar a cabo la estimación se utilizará la información histórica de las licitaciones realizadas en el SIC y SING para el suministro eléctrico de los clientes regulados, entre Octubre 2006 a Agosto 2014. La Tabla 1 resume las estadísticas descriptivas de las principales variables consideradas en el modelo econométrico presentado

\section{TABLA I. ESTADISTICAS DESCRIPTIVAS}

\begin{tabular}{|l|r|r|r|r|r|}
\hline \multicolumn{1}{|c|}{ Variable } & Obs. & \multicolumn{1}{c|}{ Media } & Desv.Est. & \multicolumn{1}{c|}{ Min } & \multicolumn{1}{c|}{ Max } \\
\hline RSI & 336 & 1.45 & 0.76 & 0.00 & 3.12 \\
Riesgo (VCmg) & 336 & 45.56 & 3.76 & 34.04 & 50.73 \\
Sistema & 336 & 0.98 & 0.13 & 0.00 & 1.00 \\
Duración & 336 & 12.93 & 1.73 & 2.00 & 15.00 \\
Tamaño & 336 & $1,989.29$ & 910.55 & 150.00 & 4545.40 \\
\hline
\end{tabular}

Cabe destacar que el cálculo de la volatilidad de los costos marginales se llevó a cabo eliminando la tendencia de la serie temporal de precios, de forma de reflejar estrictamente el "movimiento" de los precios esperados en torno a dicha tendencia. Para ello se usó el filtro de Hodrick y Prescott para extraer el componente tendencia de una serie temporal.

El índice RSI tiene un valor promedio de 1.45 entre todas las ofertas emitidas (336 en total) para cada sub-bloque licitado. Este valor se reduce a 1.1 cuando se calcula el promedio por bloque ( 24 bloques) y a 1.06 en promedio ponderado por el tamaño de cada bloque ${ }^{9}$. Awad et al [17] menciona que para el mercado de California, un RSI menor a 1.2 estaría indicando un elevado margen debido a poder de mercado. Esto indica, preliminarmente, que hay espacio para aumentar la competencia en las licitaciones reguladas. Asimismo, la varianza entre procesos y bloques es elevada, yendo desde RSI iguales a cero - lo que indica que sólo un oferente se presentó - hasta un RSI de 3.12.

La variable Sistema es igual a 1 si la observación pertenece al sistema SIC. Como puede observarse, la mayoría de las observaciones corresponde a licitaciones en el SIC. La duración promedio de los contratos es de casi 13 años. Finalmente, el tamaño promedio de los contratos es de casi 1990 GWh por año.

\section{RESULTADOS}

Para la estimación econométrica se utilizó como indicador de C (o costo competitivo) el costo de desarrollo de una central a carbón al momento de la presentación de ofertas ${ }^{10}$, ya que dicho costo refleja de hecho la mejor estimación del costo

\footnotetext{
${ }^{9}$ Las licitaciones para clientes regulados se llevan a cabo para bloques de energía en el tiempo, los cuales, para efectos de facilitar la competencia, se separan en sub-bloques al interior de cada bloque.

${ }^{10}$ Que se asume puede representar correctamente el tipo de tecnología que marcará la tendencia de las nuevas inversiones eficientes en centrales generadoras que operan en base.
}

marginal promedio del sistema, en tanto costo de proveer un contrato cualquiera.

TABLA 2. ESTIMACIÓN ECONOMÉTRICA

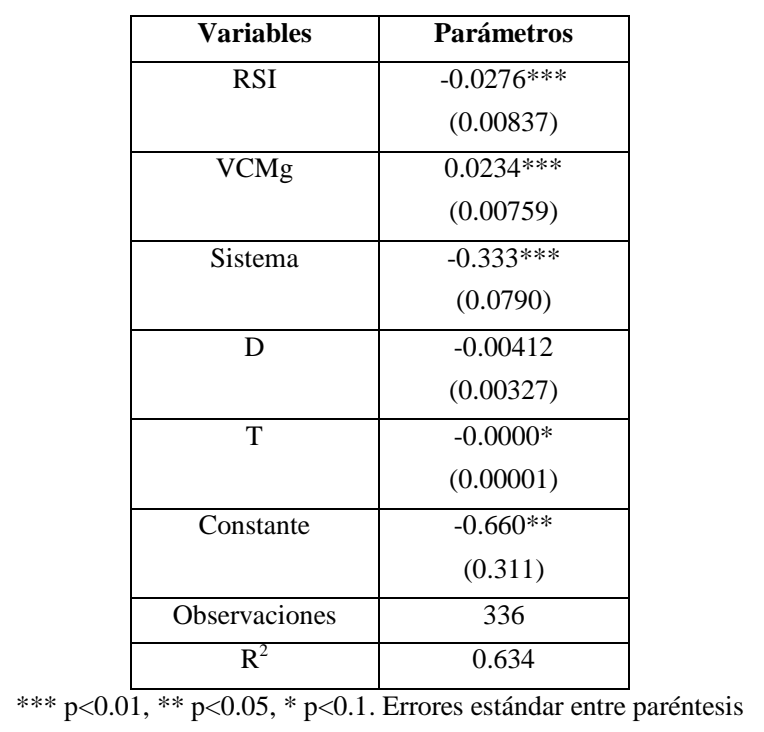

En la Tabla 2 se observa que existe evidencia de poder de mercado, significativo estadísticamente, con un coeficiente asociado a la variable RSI de valor negativo. De esta manera, si el RSI aumentara en una desviación estándar (0.76 según Tabla 1), el margen (índice de Lerner) se reduciría en 0,021 puntos.

La volatilidad de los costos marginales, indicador del riesgo de contratación, también es significativa estadísticamente. Una reducción de un punto en la desviación estándar de los costos marginales reduce el margen en 0.023 puntos. Por su parte, el tamaño y la duración del contrato de suministro no aparecen como significativos.

Es importante recalcar que dado que las licitaciones se realizan con años de antelación al inicio del contrato, lo teóricamente correcto es utilizar, como indicador de la variable "C" (costo competitivo), el costo de desarrollo o precio competitivo de largo plazo al momento de presentación de ofertas. Sin perjuicio de lo anterior, como mecanismo de control también se estimó el modelo usando el costo marginal (en vez del costo de desarrollo), contexto en el que los resultados obtenidos mantienen los signos correctos, con algunas variaciones en las magnitudes absolutas ${ }^{11}$.

\section{PROYECCIONES}

A continuación se realiza una proyección de escenarios futuros de precios al incorporarse un cambio estructural en la competencia del mercado de contratos: la interconexión de los dos mayores sistemas eléctricos del país, Sistema Interconectado Central (SIC) y Sistema Interconectado del Norte Grande (SING).

Según se calculó anteriormente, el RSI promedio ponderado para las licitaciones analizadas entre 2006 y 2014 fue de 1.06.

\footnotetext{
${ }^{11}$ Básicamente, lo que se buscó con esta variación es mostrar la robustez de la especificación del modelo, con una definición alterativa, también plausible, de una de las variables explicativas.
} 
Cabe señalar en este contexto que para el SING sólo existe una licitación en el 2009, por lo cual, a efectos de considerar el impacto de la interconexión es necesario simular un RSI una vez que los mercados se encuentren interconectados. Para ello se utilizó como punto de partida que la mayor oferta presentada y adjudicada en la única licitación realizada en el SING corresponde a una empresa sólo presente en dicho sistema. Tomando el hecho que con la interconexión al menos habría un nuevo participante, presente tanto en el SIC como en el SING, se procedió a realizar las siguientes simulaciones alternativas:

a) Simular que la empresa entrante hubiera ofrecido en los distintos bloques asociados a las licitaciones del 2006 al 2014, lo mismo que ofreció en el SING (2,300 GWh) con una probabilidad igual a la proporción de veces que se presentó una empresa incumbente de similar tamaño y portafolio de generación (valor cercano al 40\%).

b) Simular que la empresa entrante se hubiera comportado exactamente como la empresa incumbente, de similar tamaño y portafolio de generación, en cuanto a cantidad ofrecida en cada bloque.

El RSI con entrante simulado, calculado como promedio ponderado del tamaño del bloque, es igual a 1.596 en la primera simulación y 1.625 en la segunda simulación. Por lo tanto se asumirá que el RSI con interconexión será igual a 1.6. La variabilidad de precios spot sin tendencia (aplicando el filtro de Hodrick y Prescott) difiere entre sistemas. Siendo el SIC un sistema hidrotérmico, el costo marginal varía de acuerdo a la hidrología, mientras que el SING es un sistema casi completamente térmico. Por lo tanto, hay que considerar que en cuanto a riesgo, una interconexión disminuye la volatilidad de precio spot en el SIC, pero la aumenta en el SING. Para ello se utilizaron las estimaciones realizadas por la CNE en el marco del estudio del año 2014; "Evaluación de Impactos Económicos Sociales de un Proyecto de Interconexión entre los Sistemas SIC y SING”. En dicho trabajo se realizó una proyección de variabilidad y nivel de precios spot para el periodo 2014-2033, para diferentes escenarios tecnologías de expansión $^{12}$ y alternativas de interconexión. Como resultado de estas proyecciones, la desviación estándar promedio, por escenario y alternativa de interconexión, de los costos marginales esperados sería: 13.6 para el SIC, 5.6 para el SING y 8.9 para el caso con interconexión SIC-SING.

En la Tabla 3 se exhibe el impacto que tendría una interconexión si el índice RSI pasa de 1.06 a 1.6 y la volatilidad en costos marginales se reduce de 11.52 a $8.9 .^{13}$

Se puede constatar que el margen en los precios promedio de contratos regulados caería en 7.6 puntos al considerar mayor competencia y menor variabilidad de costos marginales. Dado que el margen promedio histórico en las licitaciones ha sido de $11.6 \%$ por encima del costo de desarrollo (valor promedio del índice de Lerner), si suponemos un costo de desarrollo de 90

\footnotetext{
${ }^{12}$ Carbón, ERNC, GNL, ERNC potenciado y Carbón + Hidroelectricidad

${ }^{13}$ La desviación estándar de los costos marginales sin interconexión se hizo ponderando 0.75 la volatilidad del SIC y 0.25 la volatilidad del SING. Dichos ponderadores se obtuvieron de la generación de cada sistema en el año 2014 (www.cne.cl)
}

USD/MWh, el cual puede asociarse a una central a carbón, la interconexión significaría una caída en aproximadamente 8 USD/MWh.

TABLA 3: EFECTO EN MARGEN DE CAMBIOS EN CONCENTRACIO
\begin{tabular}{|c|c|c|}
\hline Y VOLATILIDAD DE COSTOS MARGINALES \\
\hline Variables & RSI & VCmg \\
\hline $\begin{array}{c}\text { Parámetro } \\
\text { estimado }\end{array}$ & -0.0276 & 0.0234 \\
\hline $\begin{array}{c}\text { Forma de } \\
\text { medición }\end{array}$ & $\begin{array}{c}\text { Residual } \\
\text { Supply Index }\end{array}$ & $\begin{array}{c}\text { Desviación estándar } \\
\text { de serie de costos } \\
\text { marginales sin } \\
\text { tendencia }\end{array}$ \\
\hline $\begin{array}{c}\text { Valor sin } \\
\text { Interconexión }\end{array}$ & 1.06 puntos & 11.52 \\
\hline $\begin{array}{c}\text { Valor con } \\
\text { Interconexión }\end{array}$ & 1.6 puntos & 8.9 puntos \\
\hline $\begin{array}{c}\text { Efecto en el } \\
\text { mark up de } \\
\text { contratos }\end{array}$ & $\mathbf{- 0 . 0 1 5}$ & $\mathbf{- 0 . 0 6 1}$ \\
\hline
\end{tabular}

En definitiva, los resultados indican que una interconexión entre los sistemas SIC y SING genera beneficios relevantes en precios de contratos regulados, tanto por una menor concentración de la oferta como por una disminución en el riesgo de contratación.

\section{CONCLUSIONES}

El presente trabajo constituye un aporte en el diseño y medición de impactos en expansiones de redes de transmisión eléctrica. Se ha demostrado que el enfoque tradicional de calcular ahorros en costos de operación e inversión es incompleto ya que no considera el impacto sobre los precios transados en el mercado de contratos, el cual se puede medir modelando las decisiones de precios en dicho mercado.

Este trabajo propone una estimación econométrica en forma reducida, donde los precios de contratación dependen del precio spot esperado en el futuro o costo de desarrollo de largo plazo, más un margen de comercialización que es una función del poder de mercado que pueda ejercer el suministrador, el riesgo de contratación y las características propias del contrato.

A partir de datos provenientes de licitaciones públicas por suministro a clientes regulados en Chile desde el 2006 al 2014, se han estimado a través de un modelo RSI los principales determinantes de las ofertas presentadas. Las estimaciones realizadas sirvieron para proyectar los precios promedio esperados de una interconexión entre los sistemas SIC y SING, la cual constituye una obra relevante de expansión del sistema de transmisión en Chile.

Dado que un cambio relevante en la red de transmisión tiene un efecto sobre los precios de contratos de suministro, se deduce de la investigación realizada que es recomendable que en el análisis de expansión de las inversiones en transmisión se consideren los costos y beneficios económicos que involucran las mismas para el mercado de contratos. Por esta razón, se hace necesario transitar desde un modelo de decisión de inversión en transmisión basado en criterios centrados en 
los costos de operación e inversión, hacia un modelo que incluya efectos en precios de mercado, como el que se presenta en este trabajo.

El principal aporte de esta investigación radica en estimar las ganancias de una interconexión entre sistemas eléctricos poniendo el foco en el mercado de contratos. A diferencia de la metodología TEAM y otros papers similares que se encuentran en la literatura, el presente trabajo se centra en el mercado de contratos y no en precios de equilibrio en bolsas de energía.

\section{AGRADECIMIENTOS}

Agradecimientos por los comentarios recibidos de parte de los asistentes al V Encuentro Latinoamericano de Economía de la Energía, IAEE 2015, y a la Comisión Nacional Energía por la información suministrada.

\section{REFERENCIAS}

[1] Wu, F. F., Zheng, F. L., \& Wen, F. S. "Transmission investment and expansion planning in a restructured electricity market”, Energy, 31(6), 2006, pp 954-966.

[2] De Nooij, Michiel "Social cost-benefit analysis of electricity interconnector investment: A critical appraisal”, Energy Policy 39(6), 2011, pp 3096-3105

[3] S. Borenstein, J. Bushnell y S. Stoft "The Competitive Effects of Transmission Capacity in a Deregulated Electricity Industry", The RAND Journal of Economics, 31 (2), 2000, pp. 294-325.

[4] P. Joskow y J. Tirole "Transmission rights and market power on electric power networks" The Rand Journal of Economics; 31, 2000, pp. 45087.

[5] M. S. Arellano, y P. Serra "The Competitive Role of the Transmission System in Price-Regulated Power Industries," Energy Economics, vol. 30, 2008, pp 1568-1576.

[6] European Commission. Green paper. Towards a secure, sustainable and competitive European energy network. Brussels 13.11.2008. COM (2008) 782 final $\{$ SEC (2008)2869\}.

[7] CAISO "Economic evaluation of Transmission interconnection in a restructured market", informe preparado para California Energy Commission por Consortium of Electric Reliability Technology Solutions, 2004.

[8] Wolak, Frank "Valuing Transmission Investment in A Wholesale Market Regime", Presented to the California Independent System Operation TEAM Meeting, February 3, 2004.

[9] Wolak, Frank "Measuring the competitiveness benefits of a transmission investment policy: the case of the Alberta electricity market", mimeo, 2008, preparado para Alberta Electric System Operator.

[10] Sauma Enzo, Samuel Jerardino, Carlos Barria, Rodrigo Marambio, Alberto Brugman y José Mejía "Electric-systems integration in the Andes community: Opportunities and threats" Energy Policy, 39 (2), 2011, pp 936-949.

[11] Ulloa, H. "Interconexión SIC-SING y emisiones de carbono del sector eléctrico" Disertación de Doctorado, Pontificia Universidad Católica de Chile, 2012.

[12] Comisión Nacional de Energía "Impacto Económico y Social de la Interconexión SIC - SING”, Informe Final, 13 de Junio, 2013.

[13] Bustos-Salvagno J. \& F. Fuentes "Interconexión Eléctrica en Chile: precios versus costos". Documento de Investigación I - 310, Universidad Alberto Hurtado, 2015.

[14] Bustos-Salvagno J. "Bidding behavior in the Chilean Electricity Market" Serie Documento de Trabajo NEST N 7, Universidad Mayor, 2013.

[15] Fabra, Natalia, Juan Pablo Montero y Mar Reguant "La Competencia en el Mercado Eléctrico Mayorista en Chile", Informe Fiscalía Nacional Económica, 2014.

[16] Varas P. y H. Rudnick "Empirical Studies of Competition in the Chilean Electricity Market. IEEE LATIN AMERICA TRANSACTIONS, 12,8.

[17] Awad, M., K. Casey, A. Geevarghese, J. Miller, A.Rahimi, A. Sheffrin, M. Zhang, E. Toolson, G. Drayton, B. Hobbs, F. Wolak "Using market simulations for economic assessment of transmission upgrades:
Application of the California ISO approach." Restructured electric power systems: Analysis of electricity markets with equilibrium models. pp 241. 2010

[18] Fuentes, F., C. García y F. Pinto (2013). "Energía y Ciclo Económico en la Economía Chilena". Serie Documentos de Investigación, Universidad Alberto Hurtado I- 289.

Javier Bustos-Salvagno Licenciado en Economía de la Universidad Católica

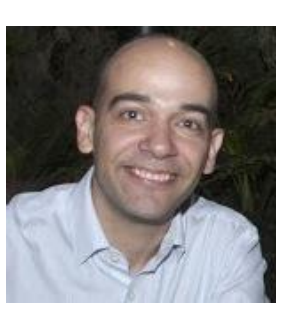
de Cuyo, Argentina, MA y Ph.D. en Economía de Georgetown University, Estados Unidos. Profesor Asociado de la Facultad de Economía y Negocios, Universidad Alberto Hurtado, Santiago, Chile. Docente e investigador en las áreas de regulación económica, economía de la energía, organización industrial y desarrollo económico. Ha sido consultor para el Banco Mundial, ONGs y el Gobierno de Chile.

Fernando Fuentes H. Ingeniero Comercial U. de Chile, MA y $\mathrm{PhD}(\mathrm{c})$ en

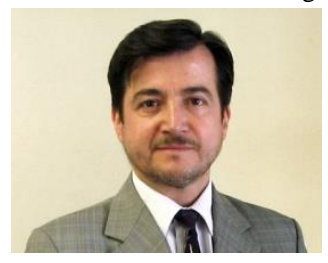
Economía Georgetown University, Magister en Filosofía de las Ciencias U. de Santiago, y Licenciado en Ciencias del Desarrollo de ILADES. Profesor e Investigador de la Facultad de Economía y Negocios Universidad Alberto Hurtado, Santiago, Chile. Docente e investigador en las áreas de regulación económica, mercados eléctricos y organización industrial. Ha sido consultor para el Banco Mundial, BID ONGs y el Gobierno de Chile. 